\title{
The Role of Jak/STAT Pathways in Osteoclast Differentiation
}

\author{
Youngkyun Lee ${ }^{1,2}$ and Hong-Hee Kim ${ }^{1, *}$ \\ ${ }^{1}$ Department of Cell and Developmental Biology, School of Dentistry, \\ ${ }^{2}$ Dental Research Institute, Seoul National University, Seoul 110-749, Republic of Korea
}

\begin{abstract}
Osteoclasts are bone-resorbing cells of monocyte/macrophage origin and are culprits of bone destruction associated with osteoporosis, rheumatoid arthritis, and cancer bone metastasis. Recent advances in osteoclast biology revealed central roles of various cytokines in regulating osteoclastogenesis both in vitro and in vivo. However, exact underlying mechanisms including signaling pathways downstream of receptor ligation are still under pursuit. In the present review, the role of Jak/STAT proteins and their regulators will be discussed in connection with osteoclastogenesis, since growing evidence indicates that a number of cytokines and growth factors utilizing Jak/STAT signaling pathways affect osteoclastogenesis. A better understanding on the role of Jak/ STAT pathways in osteoclast differentiation will not only strengthen our knowledge on osteoclast biology but also provide invaluable insights into the development of anti-resorptive strategies for treating bone-lytic diseases.
\end{abstract}

Key Words: Jak, STAT, Osteoclast, Differentiation

\section{OSTEOCLAST}

Osteoclasts are the bone-resorbing cells of monocyte/macrophage origin (Boyle et al., 2003). In mice, CD45R- CD11blow bone marrow cells were identified as osteoclast precursors at least in vitro (Jacquin et al., 2006). Mizoguchi et al. established that cell cycle arrest in osteoclast precursors is prerequisite for osteoclast differentiation (Mizoguchi et al., 2009). These cells were identified as c-Fms ${ }^{+}$(M-CSF receptor) RANKL ${ }^{+}$ cells at the site of osteoclastogenesis in vivo. Upon stimulation of these osteoclast precursors with macrophage colonystimulation factor (M-CSF) and receptor activator of nuclear factor kappa B ligand (RANKL), these cells differentiate and finally fuse to form multinuclear functional osteoclasts through a multi-step process. M-CSF supports the survival and proliferation of osteoclast precursors. The critical role of M-CSF in osteoclastogenesis was revealed by the osteopetrotic bone phenotype of op/op mice, which lack functional M-CSF leading to the absence of osteoclasts (Yoshida et al., 1990). Genetic ablation of RANKL also induced osteopetrosis accompanied by complete loss of osteoclasts (Kong et al., 1999). Ligation of RANKL receptor (RANK) by RANKL recruits TNF receptor associated factors (TRAFs) and stimulates NF- $\mathrm{KB}$, c-Fos, and NFATc1-mediated gene transcription by activating multiple signaling cascades including mitogen-activated pro-

www.biomolther.org

Open Access DOI: 10.4062/biomolther.2011.19.2.141

pISSN: 1976-9148 elSSN: 2005-4483

Copyright $\odot 2011$ The Korean Society of Applied Pharmacology tein kinases such as ERK, JNK, and p38 as well as phosphatidylinositol 3-kinase (PI3K)/Akt pathways (Lee and Kim, 2003). The major osteoclast signaling pathway is depicted in Fig. 1. Transcription factors playing critical roles also have been identified by loss of function mutation studies in mice. One of these transcription factors involved in osteoclastogenesis is hematopoietic transcription factor PU.1. Mice deficient in PU.1 developed osteopetrosis due to the loss of osteoclasts (Tondravi et al., 1997). Another family of transcription factors affecting osteoclastogenesis is microphthalmia-associated transcription factor (MITF). Mice having mutations in this transcription factor have long been known for their osteopetrotic bone phenotype (Walker, 1975). The importance of $\mathrm{NF}_{\kappa \mathrm{B}}$ in osteoclastogenesis was suggested by the study by lotsova et al., which

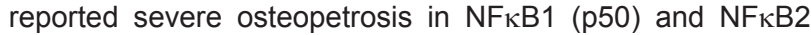
(p52) double knockout mice (lotsova et al., 1997). Mice deficient in c-Fos also developed osteopetrosis due to a lineage shift in myeloid precursors that blocked osteoclastogenesis while stimulating macrophage differentiation (Grigoriadis et al., 1994). In addition to these transcription factors, nuclear factor of activated T cells cytoplasmic 1 (NFATc1) is considered as a mater transcription factor for osteoclast terminal differentiation and function (Takayanagi et al., 2002a). Not only NFATc1-deficient cells did not differentiate into osteoclasts in response to RANKL stimulation, overexpression of NFATc1
*Corresponding Author

E-mail: hhbkim@snu.ac.kr

Tel: +82-2-740-8686, Fax: +82-2-740-8656 


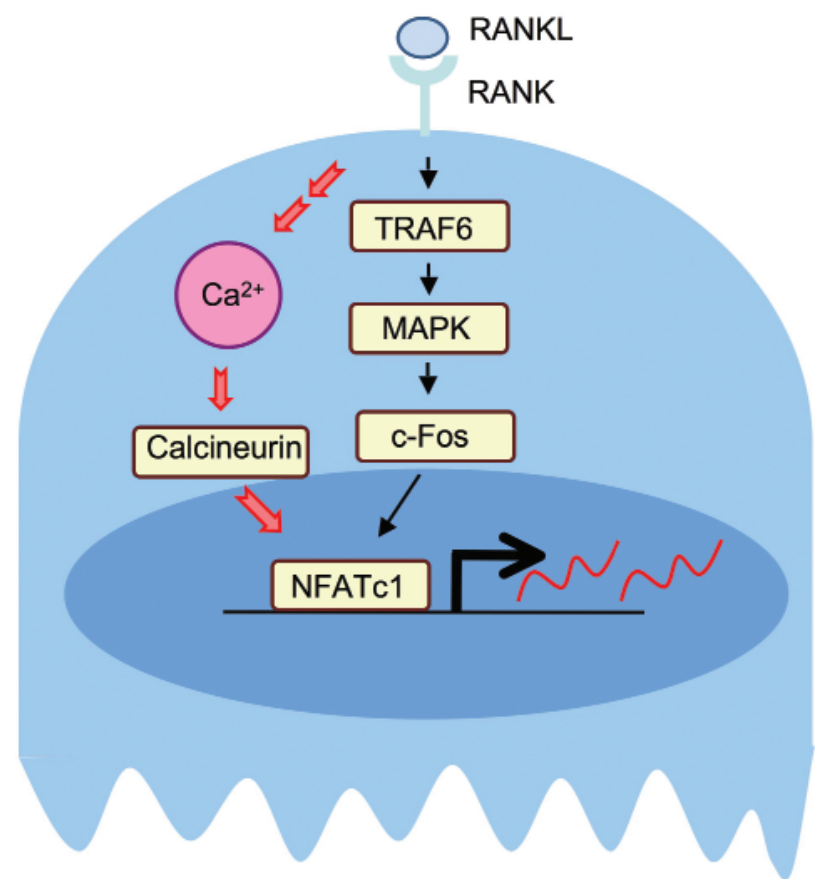

Fig. 1. Osteoclast signaling pathway. Upon RANK ligation by RANKL, TRAF is recruited followed by activation of MAPKs such as ERK, JNK, and P38. Consequently, c-Fos expression and activity is increased resulting in the induction of NFATc1. Alternatively, increased in the intracellular $\mathrm{Ca}^{2+}$ concentration upon RANKL stimulation activates $\mathrm{Ca}^{2+}$-dependent phosphatases calcineurin. Dephosphorylation by calcineurin promotes nuclear translocation of NFATc1, supporting the transcription of osteoclastogenic genes.

alone was sufficient to induce osteoclastogenesis in the absence of RANKL, suggesting that NFATc1 is both necessary and sufficient for osteoclast differentiation. Genes induced by these osteoclastogenic transcription factors include proteases, ion pumps, and membrane proteins involved in osteoclast fusion and function. For example, protease cathepsin K, produced upon MITF transactivation ( $\mathrm{Hu}$ et al., 2007), catabolizes collagen matrices to degrade bone and cartilage. A proton pump vacuolar ATPase (V-ATPase) showed significantly higher expression in mature osteoclasts than in their precursors. Interestingly, deletion of d2 subunit of V-ATPase during early stages of osteoclastogenesis resulted in defective osteoclast fusion (Lee et al., 2006). However, when depleted during later stages of osteoclast differentiation, it was evident that V-ATPase functions as proton pump that acidifies extracellular matrices during bone resorption (Wu et al., 2009). Dendritic cell-specific transmembrane protein (DC-STAMP) was identified as a protein highly expressed in osteoclast compared with its precursor (Yagi et al., 2005) and its expression was greatly induced by NFATc1 (Kim et al., 2008). In DC-STAMPdeficient mice, osteoclast fusion was completely impaired although expression of osteoclast differentiation markers was normal, suggesting that the function of DC-STAMP is cell fusion-specific (Yagi et al., 2005). Upon these concerted actions of multiple genes, mature multinucleated osteoclasts are formed. These osteoclasts undergo structural changes that allow the formation of sealing zone between bone surface and osteoclast basal membrane, and the secretion of acids as well as lytic enzymes into the lacunae leading to the bone resorption (Boyle et al., 2003).

\section{BONE-DESTRUCTIVE DISEASES AND ANTI-OSTEO- CLASTOGENESIS DRUGS}

Since bone homeostasis is maintained by bone-formation by osteoblasts and bone-degradation by osteoclasts, many of the skeletal diseases involve unregulated osteoclastogenesis that leads to excessive bone resorption (Arai et al., 1999; Teitelbaum, 2000; Boyle et al., 2003). These include osteoporosis, rheumatoid arthritis, periodontal diseases, cancer metastasis, and multiple myeloma bone diseases. Thus the inhibition of osteoclast differentiation and/or activity is expected to alleviate bone destruction associated with these conditions. Most widely used anti-osteoclastogenic drugs are bisphosphonates (Favus, 2010). This class of drugs has high affinity to bones and is ingested by bone-resorbing osteoclasts (Sato et al., 1991; Masarachia et al., 1996). Once inside the cells, bisphosphonate induces apoptosis of osteoclasts either by acting as ATP analogue (non-nitrogen containing bisphosphonates), or by inhibiting protein prenylation that is important for the survival and activity of osteoclasts (nitrogen containing bisphosphonates) (Frith et al., 1997; van Beek et al., 2003).

Another promising class of drugs targets RANKL-RANK axis. Among them is a humanized anti-RANKL antibody denosumab developed by Amgen, which is approved by FDA in 2010 for use in post-menopausal osteoporosis. This drug mimics endogenous osteoprotegerin (OPG), which binds to RANKL and blocks RANKL-dependent osteoclastogenesis.

In the last decade extensive studies on osteoclast biology as well as on the regulation of osteoclastogenesis by immune and endocrine system greatly advanced our understanding on the process of osteoclastogenesis. Upon this understanding, several drug pipelines are under clinical trials (Yasothan and Kar, 2008), and still more possible target molecules are being suggested through basic and clinical studies. Among the wide variety of mechanisms reported to govern osteoclastogenesis, the present review will focus on the Janus kinase/ signal transducers and activators of transcription (Jak/STAT) signaling pathways.

\section{THE JAK/STAT PATHWAY}

Jak/STAT signaling pathway is one of the most extensively studied signal transduction cascade in mammals. More than 30 cytokines and receptor ligands have been shown to utilize JAK/STAT pathway to integrate extracellular signal to modulate gene expression and cellular functions (Rawlings et al., 2004; Murray, 2007). These include type I and type II interferons, GP130 family cytokines, interleukins, and growth hormones. There are four Jak family members (Jak1, Jak2, Jak3, and Tyk2) (Stark et al., 1998) and seven STAT family members (STAT1, STAT2, STAT3, STAT4, STAT5a, STAT5b, and STAT6) (Darnell, 1997). The importance of Jak/STAT signaling has been underscored by embryonic or perinatal lethality or severe defects in immune system or growth hormone pathways in knockout mice (Shuai and Liu, 2003). Upon ligation, the Jak-binding receptors multimerize allowing trans-phosphorylation of Jaks (Murray, 2007). Subsequently, phosphory- 
lated active Jak kinases phosphorylate cytosolic domains of receptors that serve as docking sites for the Src homology 2 $(\mathrm{SH} 2)$ domains in STATs. Finally, STATs are phosphorylated by Jaks and other proximal kinases, dimerize, and translocate into the nucleus to regulate gene transcription. These Jak/ STAT signaling pathways are further fine-tuned by negative regulation by suppressor of cytokine signaling (SOCS) (Hilton, 1999) and protein inhibitor of activated STAT (PIAS) (Shuai, 2000).

\section{REGULATION OF OSTEOCLAST DIFFERENTIATION BY INTERFERONS}

Due to the dramatic expansion in the understanding of molecular mechanisms underlying osteoclastogenesis including regulation by immune system, a variety of cytokines has been shown to have anti-osteoclastogenic effects and suggested as anti-resorptive strategy. Among them, interferon $\gamma$ produced by $\mathrm{T}$ cells was reported to inhibit RANKL-dependent osteoclast differentiation (Takayanagi et al., 2000). Interferon $\gamma$ rapidly induced proteasome-mediated TRAF6 degradation, resulting in the loss of RANKL-stimulated NFKB and JNK activities. Accordingly, overexpression of TRAF6 in osteoclast precursors reduced the inhibitory effect of interferon $\gamma$ on osteoclastogenesis. Interferon $\beta$ added another layer of fine-tuning mechanism by which RANKL-dependent osteoclast differentiation is regulated. Takayanagi et al. reported that RANKL stimulation of osteoclast precursors rapidly induced the production of interferon $\beta$ in a c-Fos dependent mechanism (Takayanagi et al., 2002b). Interestingly, interferon $\beta$ strongly inhibited RANKL-induced osteoclastogenesis by reducing the expression of c-Fos. Indeed, both interferon $\beta$-deficient mice and interferon $\alpha / \beta$-receptor-deficient mice showed reduced bone mass with significantly increased osteoclast number. Thus, interferon $\beta$ comprised an autoregulatory negative feedback loop in which bone homeostasis might be regulated by limiting extensive bone destruction by osteoclasts.

\section{REGULATION OF OSTEOCLAST DIFFERENTIATION BY INTERLEUKINS THAT STIMULATE JAK/STAT PATHWAY}

A number of interleukins that utilize JAK/STAT signaling pathways were investigated regarding the role in regulating osteoclast differentiation. Among them, IL-6 is one of the most extensively studied. Duplomb et al. showed that IL-6 potently inhibited RANKL-induced osteoclastogenesis of mouse macrophages as well as human monocytes in a STAT3 dependent fashion (Duplomb et al., 2008). IL-6 diverted osteoclast precursors into macrophages in the presence of RANKL in RAW 264.7 cells, mouse bone marrow macrophages, and human peripheral blood monocytes. Yoshitake et al. showed that IL-6 also inhibited RANKL-induced NFKB and MAPK signaling pathways in mouse bone marrow macrophages (Yoshitake et al., 2008). On the other hand, IL-6 stimulated the formation of osteoclasts when added to osteoblast/osteoclast co-cultures (Tamura et al., 1993; Richards et al., 2000). This indirect stimulation of osteoclastogenesis might have resulted from the increased production of RANKL by osteoblasts in the presence of IL-6 (Palmqvist et al., 2002). For the augmented osteoclastogenesis by osteoblasts in the presence of IL-6, the activity of STAT3 was crucial since no increase in the RANKL in osteoblasts was observed when dominant-negative form of STAT3 was overexpressed (O'Brien et al., 1999).

IL-4 has been shown to suppress osteoclast differentiation via STAT6 by several independent research groups. Abu-Amer was one of the researchers first described the inhibitory role of IL-4 on osteoclast differentiation (Abu-Amer, 2001). IL-4 significantly reduced RANKL-dependent NFKB activation and completely inhibited osteoclastogenesis in mouse bone marrow macrophages. However, this negative regulation of osteoclastogenesis was not observed when bone marrow macrophages from STAT6-deficient mice were used. Moreno et al. showed that IL-4 reduced the expression of RANK mRNA, thereby reducing osteoclastogenesis in human peripheral blood monocytes and mouse bone marrow macrophages (Moreno et al., 2003). These effects were STAT6-dependent, since IL-4 did not reduce RANK expression in STAT6-deficient osteoclast precursors. Yamada et al. showed that IL-4 inhibited osteoclastogenesis in mouse bone marrow macrophages while stimulating STAT6 phosphorylation and reducing NFATc1 expression (Yamada et al., 2007). Wei et al. reported that IL-4

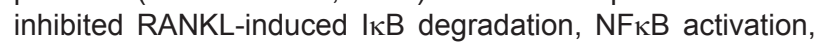
and MAPK activation in a STAT6-dependent manner in mouse bone marrow macrophages (Wei et al., 2002). IL-4 also inhibited the bone-resorbing activity of mature osteoclasts by reducing the RANKL-stimulated $\mathrm{NF} \kappa \mathrm{B}$ and $\mathrm{Ca}^{2+}$ responses (Mangashetti et al., 2005). In addition, IL-4 stimulated the production of osteoprotegerin (OPG), a decoy receptor for RANKL, in mouse osteoblasts (Yamada et al., 2007).

Direct addition of IL-7 to mouse bone marrow cells significantly suppressed osteoclast formation in the presence of M-CSF and RANKL suggesting a negative role of this interleukin on osteoclastogenesis, which was further supported by enhanced osteoclast differentiation from IL-7-deficient bone marrow cells (Lee et al., 2003). However, Weitzmann et al. reported a dramatic increase in the osteoclast differentiation from human peripheral blood mononuclear cells by IL-7 (Weitzmann et al., 2000). In this case, the stimulatory effect of IL-7 was mediated via up-regulation of RANKL expression in $\mathrm{T}$ cells.

IL-15 stimulated the formation of osteoclasts from rat whole bone marrow cells as well as stroma-depleted marrow cells following vitamin $D_{3}$ addition (Ogata et al., 1999). In support of the pro-osteoclastogenic role of IL-15, recent report by Djaafar et al. showed significantly increased bone mass in IL15 receptor-deficient mice (Djaafar et al., 2010). Interestingly, IL-15 receptor-deficient T cells showed impaired expression of RANKL and failed to support wild type osteoclasts, while IL-15 receptor-deficient osteoclast precursors did not form mature osteoclasts even in the presence of wild type $T$ cell. These results suggested the existence of both direct and indirect inhibitory mechanisms on osteoclastogenesis by IL-15.

IL-12 has been known to induce Jak2- and Tyk2-dependent STAT phosphorylation (Zou et al., 1997). IL-12 inhibited osteoclastogenesis from murine spleen cells as well as osteoblast/ splenocyte co-cultures (Horwood et al., 2001). These inhibitory effects almost completely disappeared when $\mathrm{T}$ cells were removed from the spleen cell cultures, suggesting that $T$ cells mediated the IL-12-dependent suppression of osteoclastogenesis. However, contradictory results were obtained when Nagata et al. treated mouse bone marrow macrophages with IL-12 (Nagata et al., 2003). Although IL-12 had no effect on the 
differentiation of osteoclast from bone marrow macrophages stimulated by M-CSF and RANKL, IL-12 potently inhibited osteoclastogenesis when mouse whole bone marrow cells were used as osteoclast precursors. In addition, this inhibitory effect of IL-12 appeared to be mediated by interferon $\gamma$, since the addition of anti-interferon $\gamma$ antibody blunted the suppression of osteoclastogenesis by IL-12. In contrast to the results by Horwood et al. (2001). IL-12 still potently blocked the formation of osteoclastogenesis from bone marrow cells even after $T$ cell depletion or when bone marrow cells from $T$ cell-deficient nude mice were used. On the other hand, B cell depletion slightly reduced the inhibitory effect of IL-12 on osteoclast differentiation, suggesting an involvement of B cells.

$\mathrm{IL}-23$ is a heterodimeric cytokine sharing a subunit of IL12 (Lankford and Frucht, 2003). Both inhibitory and stimulatory roles of IL-23 on osteoclastogenesis through T cells were reported. Quinn et al. reported that IL-23 inhibited osteoclast formation from mouse spleen cells in $\mathrm{CD}^{+} \mathrm{T}$ - and $\gamma \delta \mathrm{T}$ celldependent mechanism (Quinn et al., 2008). Mice lacking a subunit of IL-23 (IL-23p19 deficiency) represented reduced bone mass phenotype, supporting the role of IL-23 in limiting bone resorption in resting conditions. In contrast, Ju et al. reported that IL-23 stimulated the expression of RANKL on CD4 ${ }^{+}$ $T$ cells from arthritic IL-1 receptor antagonist (IL-1Ra)-deficient mice, enhancing osteoclastogenesis (Ju et al., 2008). However, these authors could not observed the IL-23-dependent augmentation of osteoclast differentiation when wild type bone marrow cells were used, suggesting that the inflammatory condition affected the effects of IL-23 on osteoclastogenesis.

IL-3 directly inhibited osteoclast differentiation from human peripheral blood monocytes (Gupta et al., 2010). The addition of IL-3 greatly suppressed the expressions of c-Fms, PU.1, and c-Fos. Interestingly, IL-3 not only inhibited osteoclasto- genesis, but also diverted osteoclast precursors into dendritic cell-like phenotype. IL-3 also suppressed osteoclastogenesis from mouse bone marrow macrophages (Khapli et al., 2003). In this setting, IL-3 inhibited IкB phosphorylation, NFKB nuclear translocation, and c-Fos transcription upon RANKL stimulation of osteoclast precursors. In contrast to the results in human cells, IL-3 increased the expression of macrophage markers such as Mac-1 and F4/80.

IL-10 suppressed RANKL-dependent osteoclast differentiation from RAW264.7 macrophages as well as mouse bone marrow cells by reducing the expression of c-Fos, c-Jun, and NFATc1 (Mohamed et al., 2007). Similar inhibitory role of IL-10 on osteoclastogenesis was reported when mouse osteoblast/ bone marrow cell co-culture system was used (Hong et al., 2000).

Both leukemia inhibitory factor (LIF) and oncostatin M (OSM) stimulated osteoclast formation by stimulating RANKL expression in mouse calvariae (Palmqvist et al., 2002). Similarly, IL-11 induced RANKL expression in cultured mouse calvarial bones and stimulated bone resorption (Ahlen et al., 2002). The effect of various interleukins on osteoclastogenesis is summarized in Table 1.

\section{ROLE OF JAK FAMILY PROTEINS}

In spite of the vast amount of evidence suggesting that interferons and interleukins that stimulate Jak/STAT pathways affect the differentiation of osteoclasts and bone homeostasis, little is known on the role of Jak family kinases in these processes. We previously showed that the expression of Jak1 significantly decreased during RANKL-induced osteoclast differentiation of mouse bone marrow macrophages, while other

Table 1. Effect of selective interleukins on osteoclastogenesis

\begin{tabular}{clll}
\hline Interleukin & \multicolumn{1}{c}{ Cell } & Effect & \multicolumn{1}{c}{ Reference } \\
\hline IL-6 & Mouse BMM, Human Monocytes & Inhibition & Duplomb et al., 2008 \\
& Mouse BMM & Inhibition & Yoshitake et al., 2008 \\
& OB/OC co-culture & Stimulation & Tamura et al., 1993; Richards et al., 2000 \\
IL-4 & Mouse BMM & Inhibition & Abu-Amer, 2001 \\
& Human PBMC, Mouse BMM & Inhibition & Moreno et al., 2003 \\
& Mouse BMM & Inhibition & Yamada et al., 2007 \\
IL-7 & Mouse BMM & Inhibition & Wei et al., 2002 \\
& Mouse BMM & Inhibition & Lee et al., 2003 \\
IL-15 & Human PBMC & Stimulation & Weitzmann et al., 2000 \\
IL-12 & Rat bone marrow & Mouse splenocyte & Ogata et al., 1999 \\
& Mouse bone marrow & Inhibition & Horwood et al., 2001 \\
IL-23 & Mouse splenocyte & Inhibition & Nagata et al.., 2003 \\
IL-3 & Human PBMC & Inhibition & Quinn et al., 2008 \\
& Mouse BMM & Inhibition & Gupta et al., 2010 \\
IL-10 & RAW264.7, Mouse bone marrow & Inhibition & Khapli et al., 2003 \\
& Mouse OB/bone marrow co-culture & Inhibition & Mohamed et al., 2007 \\
LIF & Mouse calvaria & Inhibition & Hong et al., 2000 \\
OSM & Mouse calvaria & Stimulation & Palmqvist et al.,, 2002 \\
IL-11 & Mouse calvaria & Stimulation & Palmqvist et al., 2002 \\
\hline
\end{tabular}


Jak family members such as Jak2 and Jak3 did not change in protein level (Lee et al., 2008). The reduction of Jak1 expression was mediated by ubiquitination followed by proteasomedependent degradation. The reduction in Jak1 expression during osteoclast differentiation was associated with reduced susceptibility to the inhibition by interferon $\beta$. Although interferon $\beta$ potently suppressed osteoclastogenesis in osteoclast precursors when Jak1 protein is in normal levels, interferon $\beta$ lost its inhibitory effects especially in the later phase of differentiation, when Jak1 expression was significantly diminished. In addition, the Jak1-dependent inhibition of osteoclastogenesis was mediated by STAT3. Compared with STAT1 and STAT5, only the phosphorylation of STAT3 was impaired in Jak1 knocked down cells as well as in RANKL-primed osteoclast precursors in which Jak1 expression was decreased. In addition, interferon $\beta$ did not inhibit osteoclast differentiation when STAT3 was knocked down.

Possible role of Jak2 in osteoclast survival was suggested since the incubation of osteoclasts derived from mouse bone marrow macrophages with Jak2 inhibitor AG490 resulted in the inhibition of osteoclast apoptosis upon RANKL removal (Kwak et al., 2008). However, since the specificity of AG490 for Jak2 over other kinases are doubted (Osherov et al., 1993; Kleinberger-Doron et al., 1998), the role of Jak2 in this process requires further corroboration by alterative methods such as overexpression of dominant negative form or gene silencing.

\section{ROLE OF STAT FAMILY PROTEINS}

The role of STAT1 in osteoclastogenesis was highlighted by initial descriptions that STAT1 mediated the inhibition of osteoclast differentiation by interferon $\gamma$ (Takayanagi et al., 2000) and interferon $\beta$ (Hayashi et al., 2002). STAT1-deficient osteoclast precursors were protected from the inhibitory effects of interferon $\gamma$. Interferon $\beta$ failed to stimulate STAT1 phosphorylation and DNA binding activity in RANKL-primed osteoclast precursors that were resistant to the inhibition by this type I interferon. However, discrepant data were reported regarding the involvement of STAT1 in these processes. Huang et al. published that the incubation of osteoclast precursors with RANKL rendered resistance to interferon $\gamma$ irrespective of STAT1 phosphorylation (Huang et al., 2003). In their experiments, interferon $\gamma$ still potently induced STAT1 phosphorylation in RANKL-primed osteoclast precursors that were clearly protected from the inhibitory effect of interferon $\gamma$ on osteoclastogenesis. Similarly, we observed comparable phosphorylation of STAT1 in bone marrow macrophages upon interferon $\beta$ stimulation both before and after RANKL priming for 2 days (Lee et al., 2008). Indeed the interferon $\beta$ inhibition of osteoclastogenesis was mediated by STAT3 in these experiments. These discrepancies suggest the possibility that there might be multiple STAT signaling pathways governing the interferon-dependent regulation of osteoclastogenesis. In addition, STAT1 is a negative regulator of osteoblast differentiation by retaining runx 2 in cytoplasm through physical interaction, which is independent of STAT1 transcription activity (Kim et al., 2003).

The role of STAT3 in osteoclastogenesis is emphasized by the osteoporotic bone phenotype of mice deficient in STAT3 in hematopoietic cells (Zhang et al., 2005). In these mice, os- teoclastogenesis was dramatically increased with enhanced c-Fos expression in osteoclast precursors, suggesting an inhibitory role of STAT3 in osteoclast differentiation.

STAT6 has been associated with the IL-4-dependent inhibition of osteoclastogenesis (Abu-Amer, 2001; Wei et al., 2002; Moreno et al., 2003). In addition, constitutive active STAT6 potently inhibited RANKL-stimulated NFKB and JNK activities as well as osteoclast differentiation (Hirayama et al., 2005). Besides, STAT6 was also involved in the production of OPG in IL-4-stimulated osteoblasts, further suppressing osteoclastogenesis (Palmqvist et al., 2006; Yamada et al., 2007).

\section{ROLE OF STAT-REGULATING PROTEINS}

Suppressor of cytokine signaling (SOCS) family members are STAT-induced STAT inhibitors (Alexander, 2002). SOCS proteins either bind to phosphorylated Jaks or activated receptors to block Jak activities. It was reported that RANKL induced the expression of SOCS1 and SOCS3 in bone marrow macrophages (Hayashi et al., 2002). These STAT inhibitors were suggested to play crucial roles in the protection of RANKL-primed osteoclast precursors from the inhibition of osteoclastogenesis by endogenous interferon $\beta$. Indeed, retrovirus-mediated overexpression of SOCS3 in mouse bone marrow macrophages resulted in significantly enhanced RANKL-dependent osteoclast differentiation (Fox et al., 2003). Furthermore, SOCS3 overexpression rendered formation of mature osteoclasts in the presence of interferon $\beta$ that otherwise suppressed osteoclastogenesis completely. However, Ohishi et al. reported that the overexpression of SOCS1, not that of SOCS3, rescued osteoclastogenesis from mouse bone marrow macrophages in the presence of interferon $\gamma$ and interferon $\beta$ (Ohishi et al., 2005). These authors further showed that SOCS3-deficient osteoclast precursors were hypersensitive to the inhibitory effect of IL-6 on osteoclastogenesis, while exhibited no difference to that by both type I and type II interferons.

Protein inhibitor of activated STAT (PIAS) family members block STAT-dependent transcription either by directly inhibiting DNA-binding activity or acting as co-repressors (Chung et al., 1997; Liu et al., 1998; Liu et al., 2001; Arora et al., 2003). Among the four PIAS family members (PIAS1, PIAS3, PIASX, PIASY), only PIAS3 has been associated with osteoclastogenesis until present. Hikata et al. showed that PIAS-transgenic mice exhibited osteopetrotic bone phenotype with impaired osteoclast differentiation, suggesting that PIAS3 acted as a negative regulator of osteoclastogenesis (Hikata et al., 2009). Overexpression of PIAS3 in RAW264.7 macrophages significantly reduced osteoclastogenesis and suppressed the expression of c-Fos upon RANKL stimulation, supporting that PIAS3 directly inhibited osteoclastogenesis from precursor cells. Kim et al. reported that PIAS3 recruited a transcription co-repressor HDAC1 to NFATc1 promoter, thereby reducing NFATc1 expression and osteoclast differentiation upon RANKL stimulation of mouse bone marrow macrophages (Kim et al., 2007). However, although PIAS3 has been reported to directly bind STAT3 (Chung et al., 1997), the role of STAT3 in the PIAS3-mediated inhibition of RANKL-dependent osteoclastogenesis has not been tested in these studies and needs to be unveiled. 


\section{CONCLUSION}

Growing evidence indicates that Jak/STAT pathways are playing central roles in the regulation of immune responses. During the last decade, enormous data were gathered on the genetic, biochemical, and cell biological aspects on the regulation of osteoclast differentiation and function both in vivo and in vitro. Current understanding on the osteoclast biology suggests that immune system, endocrine system, and bone are closely related. Especially, the role of cytokines on osteoclastogenesis is under active investigation. This review focused on the studies regarding the role of Jak/STAT signaling pathways on osteoclastogenesis performed mostly in last 10 years. However, in spite of extensive research, our molecular understanding on the exact nature and the role of each component of Jak/STAT pathway is limited. Furthermore, the information on the role of cytokine/receptor/Jak/STAT combination in the context of osteoclastogenesis is lacking. Recent advances in genomic and proteomic methodology will definitely be of great help to decipher the quandary. In addition, the application of cell-specific or conditional transgenic technology will also substantially advance our knowledge on the role of Jak/STAT pathways on osteoclastogenesis in vivo, which was hindered by lethality of mice generated by systemic knock out technology.

\section{ACKNOWLEDGMENTS}

This work was supported by the $21 \mathrm{C}$ Frontier Functional Proteomics Project (FPR08B1-170, H-H. K.), Science Research Center (2010-0001744, H-H. K.), and National Research Foundation of Korea grant funded by the Korean Government, Ministry of Education, Science, and Technology (NRF-2010-359-E00016, Y. L.).

\section{REFERENCES}

Abu-Amer, Y. (2001) IL-4 abrogates osteoclastogenesis through STAT6-dependent inhibition of NF-kappaB. J. Clin. Invest. 107, 1375-1385.

Ahlen, J., Andersson, S., Mukohyama, H., Roth, C., Backman, A., Conaway, H. H. and Lerner, U. H. (2002) Characterization of the bone-resorptive effect of interleukin-11 in cultured mouse calvarial bones. Bone 31, 242-251.

Alexander, W. S. (2002) Suppressors of cytokine signalling (SOCS) in the immune system. Nat. Rev. Immunol. 2, 410-416.

Arai, F., Miyamoto, T., Ohneda, O., Inada, T., Sudo, T., Brasel, K., Miyata, T., Anderson, D. M. and Suda, T. (1999) Commitment and differentiation of osteoclast precursor cells by the sequential expression of c-Fms and receptor activator of nuclear factor kappaB (RANK) receptors. J. Exp. Med. 190, 1741-1754.

Arora, T., Liu, B., He, H., Kim, J., Murphy, T. L., Murphy, K. M., Modlin, R. L. and Shuai, K. (2003) PIASx is a transcriptional co-repressor of signal transducer and activator of transcription 4. J. Biol. Chem. 278, 21327-21330.

Boyle, W. J., Simonet, W. S. and Lacey, D. L. (2003) Osteoclast differentiation and activation. Nature 423, 337-342.

Chung, C. D., Liao, J., Liu, B., Rao, X., Jay, P., Berta, P. and Shuai, K. (1997) Specific inhibition of Stat3 signal transduction by PIAS3. Science 278, 1803-1805

Darnell, J. E. Jr. (1997) STATs and gene regulation. Science 277 1630-1635.

Djaafar, S., Pierroz, D. D., Chicheportiche, R., Zheng, X. X., Ferrari, S.
L. and Ferrari-Lacraz, S. (2010) Inhibition of T cell-dependent and RANKL-dependent osteoclastogenic processes associated with high levels of bone mass in interleukin-15 receptor-deficient mice. Arthritis. Rheum. 62, 3300-3310.

Duplomb, L., Baud'huin, M., Charrier, C., Berreur, M., Trichet, V., Blanchard, F. and Heymann, D. (2008) Interleukin-6 inhibits receptor activator of nuclear factor kappaB ligand-induced osteoclastogenesis by diverting cells into the macrophage lineage: key role of Serine727 phosphorylation of signal transducer and activator of transcription 3. Endocrinology 149, 3688-3697.

Favus, M. J. (2010) Bisphosphonates for osteoporosis. N. Engl. J. Med. 363, 2027-2035.

Fox, S. W., Haque, S. J., Lovibond, A. C. and Chambers, T. J. (2003) The possible role of TGF-beta-induced suppressors of cytokine signaling expression in osteoclast/macrophage lineage commitment in vitro. J. Immunol. 170, 3679-3687.

Frith, J. C., Monkkonen, J., Blackburn, G. M., Russell, R. G. and Rogers, M. J. (1997) Clodronate and liposome-encapsulated clodronate are metabolized to a toxic ATP analog, adenosine 5'-(beta, gamma-dichloromethylene) triphosphate, by mammalian cells in vitro. J. Bone Miner Res. 12, 1358-1367.

Grigoriadis, A. E., Wang, Z. Q., Cecchini, M. G., Hofstetter, W., Felix, R., Fleisch, H. A. and Wagner, E. F. (1994) c-Fos: a key regulator of osteoclast-macrophage lineage determination and bone remodeling. Science 266, 443-448.

Gupta, N., Barhanpurkar, A. P., Tomar, G. B., Srivastava, R. K., Kour, S., Pote, S. T., Mishra, G. C. and Wani, M. R. (2010) IL-3 inhibits human osteoclastogenesis and bone resorption through downregulation of c-Fms and diverts the cells to dendritic cell lineage. J. Immunol. 185, 2261-2272.

Hayashi, T., Kaneda, T., Toyama, Y., Kumegawa, M. and Hakeda, Y. (2002) Regulation of receptor activator of NF-kappa B ligandinduced osteoclastogenesis by endogenous interferon-beta (INFbeta ) and suppressors of cytokine signaling (SOCS) The possible counteracting role of SOCSs- in IFN-beta-inhibited osteoclast formation. J. Biol. Chem. 277, 27880-27886.

Hikata, T., Takaishi, H., Takito, J., Hakozaki, A., Furukawa, M., Uchikawa, S., Kimura, T., Okada, Y., Matsumoto, M., Yoshimura, A., Nishimura, R., Reddy, S. V., Asahara, H. and Toyama, Y. (2009) PIAS3 negatively regulates RANKL-mediated osteoclastogenesis directly in osteoclast precursors and indirectly via osteoblasts. Blood 113, 2202-2212.

Hilton, D. J. (1999) Negative regulators of cytokine signal transduction. Cell Mol. Life Sci. 55, 1568-1577.

Hirayama, T., Dai, S., Abbas, S., Yamanaka, Y. and Abu-Amer, Y. (2005) Inhibition of inflammatory bone erosion by constitutively active STAT-6 through blockade of JNK and NF-kappaB activation. Arthritis. Rheum. 52, 2719-2729.

Hong, M. H., Williams, H., Jin, C. H. and Pike, J. W. (2000) The inhibitory effect of interleukin-10 on mouse osteoclast formation involves novel tyrosine-phosphorylated proteins. J. Bone Miner Res. 15, 911-918.

Horwood, N. J., Elliott, J., Martin, T. J. and Gillespie, M. T. (2001) IL12 alone and in synergy with IL-18 inhibits osteoclast formation in vitro. J. Immunol. 166, 4915-4921.

Hu, R., Sharma, S. M., Bronisz, A., Srinivasan, R., Sankar, U. and Ostrowski, M. C. (2007) Eos, MITF, and PU.1 recruit corepressors to osteoclast-specific genes in committed myeloid progenitors. Mol. Cell Biol. 27, 4018-4027.

Huang, W., O'Keefe, R. J. and Schwarz, E. M. (2003) Exposure to receptor-activator of NFkappaB ligand renders pre-osteoclasts resistant to IFN-gamma by inducing terminal differentiation. Arthritis. Res. Ther. 5, R49-59.

lotsova, V., Caamano, J., Loy, J., Yang, Y., Lewin, A. and Bravo, R. (1997) Osteopetrosis in mice lacking NF-kappaB1 and NF-kappaB2. Nat. Med. 3, 1285-1289.

Jacquin, C., Gran, D. E., Lee, S. K., Lorenzo, J. A. and Aguila, H. L. (2006) Identification of multiple osteoclast precursor populations in murine bone marrow. J. Bone Miner Res. 21, 67-77.

Ju, J. H., Cho, M. L., Moon, Y. M., Oh, H. J., Park, J. S., Jhun, J. Y., Min, S. Y., Cho, Y. G., Park, K. S., Yoon, C. H., Min, J. K., Park, S. H., Sung, Y. C. and Kim, H. Y. (2008) IL-23 induces receptor activa- 
tor of NF-kappaB ligand expression on CD4+ T cells and promotes osteoclastogenesis in an autoimmune arthritis model. J. Immunol. 181, 1507-1518.

Khapli, S. M., Mangashetti, L. S., Yogesha, S. D. and Wani, M. R. (2003) IL-3 acts directly on osteoclast precursors and irreversibly inhibits receptor activator of NF-kappa B ligand-induced osteoclast differentiation by diverting the cells to macrophage lineage. J. Immunol. 171, 142-151.

Kim, K., Lee, J., Kim, J. H., Jin, H. M., Zhou, B., Lee, S. Y. and Kim, N. (2007) Protein inhibitor of activated STAT 3 modulates osteoclastogenesis by down-regulation of NFATc1 and osteoclast-associated receptor. J. Immunol. 178, 5588-5594.

Kim, K., Lee, S. H., Kim J. H., Choi, Y. and Kim, N. (2008) NFATc1 induces osteoclast fusion via up-regulation of Atp6v0d2 and the dendritic cell-specific transmembrane protein (DC-STAMP). Mol Endocrinol. 22, 176-185

Kim, S., Koga, T., Isobe, M., Kern, B. E., Yokochi, T., Chin, Y. E., Karsenty, G., Taniguchi, T. and Takayanagi, H. (2003) Stat1 functions as a cytoplasmic attenuator of Runx2 in the transcriptiona program of osteoblast differentiation. Genes. Dev. 17, 1979-1991.

Kleinberger-Doron, N., Shelah, N., Capone, R., Gazit, A. and Levitzki, A. (1998) Inhibition of Cdk2 activation by selected tyrphostins causes cell cycle arrest at late G1 and S phase. Exp. Cell Res. 241, 340-351.

Kong, Y. Y., Yoshida, H., Sarosi, I., Tan, H. L., Timms, E., Capparelli, C., Morony, S., Oliveira-dos-Santos, A. J., Van, G., Itie, A., Khoo W., Wakeham, A., Dunstan, C. R., Lacey, D. L., Mak, T. W., Boyle, W. J. and Penninger, J. M. (1999) OPGL is a key regulator of osteoclastogenesis, lymphocyte development and lymph-node organogenesis. Nature $397,315-323$

Kwak, H. B., Sun, H. M., Ha, H., Lee, J. H., Kim, H. N. and Lee, Z. H. (2008) AG490, a Jak2-specific inhibitor, induces osteoclast surviva by activating the Akt and ERK signaling pathways. Mol. Cells 26 436-442.

Lankford, C. S. and Frucht, D. M. (2003) A unique role for IL-23 in promoting cellular immunity. J. Leukoc. Biol. 73, 49-56.

Lee, S. H., Rho, J., Jeong, D., Sul, J. Y., Kim, T., Kim, N., Kang, J. S. Miyamoto, T., Suda, T., Lee, S. K., Pignolo, R. J., Koczon-Jaremko, B., Lorenzo, J. and Choi, Y. (2006) v-ATPase V0 subunit d2-deficient mice exhibit impaired osteoclast fusion and increased bone formation. Nat. Med. 12, 1403-1409.

Lee, S. K., Kalinowski, J. F., Jastrzebski, S. L., Puddington, L. and Lorenzo, J. A. (2003) Interleukin-7 is a direct inhibitor of in vitro osteoclastogenesis. Endocrinology 144, 3524-3531.

Lee, Y., Hyung, S. W., Jung, H. J., Kim, H. J., Staerk, J., Constantinescu, S. N., Chang, E. J., Lee, Z. H., Lee, S. W. and Kim, H $H$. (2008) The ubiquitin-mediated degradation of Jak1 modulates osteoclastogenesis by limiting interferon-\{beta\}-induced inhibitory signaling. Blood 111, 885-893.

Lee, Z. H. and Kim, H. H. (2003) Signal transduction by receptor activator of nuclear factor kappa B in osteoclasts. Biochem. Biophys. Res. Commun. 305, 211-214.

Liu, B., Gross, M., ten Hoeve, J. and Shuai, K. (2001) A transcriptional corepressor of Stat1 with an essential LXXLL signature motif. Proc. Natl. Acad. Sci. USA 98, 3203-3207.

Liu, B., Liao, J., Rao, X., Kushner, S. A., Chung, C. D., Chang, D. D and Shuai, K. (1998) Inhibition of Stat1-mediated gene activation by PIAS1. Proc. Natl. Acad. Sci. USA 95, 10626-10631.

Mangashetti, L. S., Khapli, S. M. and Wani, M. R. (2005) IL-4 inhibits bone-resorbing activity of mature osteoclasts by affecting NF-kappa B and Ca2+ signaling. J. Immunol. 175, 917-925.

Masarachia, P., Weinreb, M., Balena, R. and Rodan, G. A. (1996) Comparison of the distribution of $3 \mathrm{H}$-alendronate and $3 \mathrm{H}$-etidronate in rat and mouse bones. Bone 19, 281-290.

Mizoguchi, T., Muto, A., Udagawa, N., Arai, A., Yamashita, T., Hosoya, A., Ninomiya, T., Nakamura, H., Yamamoto, Y., Kinugawa, S., Nakamura, M., Nakamichi, Y., Kobayashi, Y., Nagasawa, S., Oda, K. Tanaka, H., Tagaya, M., Penninger, J. M., Ito, M. and Takahashi, N. (2009) Identification of cell cycle-arrested quiescent osteoclast precursors in vivo. J. Cell Biol. 184, 541-554

Mohamed, S. G., Sugiyama, E., Shinoda, K., Taki, H., Hounoki, H., Abdel-Aziz, H. O., Maruyama, M., Kobayashi, M., Ogawa, H. and
Miyahara, T. (2007) Interleukin-10 inhibits RANKL-mediated expression of NFATc1 in part via suppression of c-Fos and c-Jun in RAW264.7 cells and mouse bone marrow cells. Bone 41, 592-602.

Moreno, J. L., Kaczmarek, M., Keegan, A. D. and Tondravi, M. (2003) IL-4 suppresses osteoclast development and mature osteoclast function by a STAT6-dependent mechanism: irreversible inhibition of the differentiation program activated by RANKL. Blood 102 , 1078-1086.

Murray, P. J. (2007) The JAK-STAT signaling pathway: input and output integration. J. Immunol. 178, 2623-2629.

Nagata, N., Kitaura, H., Yoshida, N. and Nakayama, K. (2003) Inhibition of RANKL-induced osteoclast formation in mouse bone marrow cells by IL-12: involvement of IFN-gamma possibly induced from non-T cell population. Bone 33, 721-732.

O'Brien, C. A., Gubrij, I., Lin, S. C., Saylors, R. L. and Manolagas, S. C. (1999) STAT3 activation in stromal/osteoblastic cells is required for induction of the receptor activator of NF-kappaB ligand and stimulation of osteoclastogenesis by gp130-utilizing cytokines or interleukin-1 but not 1,25-dihydroxyvitamin D3 or parathyroid hormone. J. Biol. Chem. 274, 19301-19308.

Ogata, Y., Kukita, A., Kukita, T., Komine, M., Miyahara, A., Miyazaki, S. and Kohashi, O. (1999) A novel role of IL-15 in the development of osteoclasts: inability to replace its activity with IL-2. J. Immunol. 162, 2754-2760

Ohishi, M., Matsumura, Y., Aki, D., Mashima, R., Taniguchi, K., Kobayashi, T., Kukita, T., Iwamoto, Y. and Yoshimura, A. (2005) Suppressors of cytokine signaling- 1 and -3 regulate osteoclastogenesis in the presence of inflammatory cytokines. J. Immunol. 174, 3024-3031.

Osherov, N., Gazit, A., Gilon, C. and Levitzki, A. (1993) Selective inhibition of the epidermal growth factor and HER2/neu receptors by tyrphostins. J. Biol. Chem. 268, 11134-11142.

Palmqvist, P., Lundberg, P., Persson, E., Johansson, A., Lundgren, I., Lie, A., Conaway, H. H. and Lerner, U. H. (2006) Inhibition of hormone and cytokine-stimulated osteoclastogenesis and bone resorption by interleukin- 4 and interleukin-13 is associated with increased osteoprotegerin and decreased RANKL and RANK in a STAT6-dependent pathway. J. Biol. Chem. 281, 2414-2429.

Palmqvist, P., Persson, E., Conaway, H. H. and Lerner, U. H. (2002) IL-6, leukemia inhibitory factor, and oncostatin $\mathrm{M}$ stimulate bone resorption and regulate the expression of receptor activator of NFkappa B ligand, osteoprotegerin, and receptor activator of NF-kappa B in mouse calvariae. J. Immunol. 169, 3353-3362.

Quinn, J. M., Sims, N. A., Saleh, H., Mirosa, D., Thompson, K., Bouralexis, S., Walker, E. C., Martin, T. J. and Gillespie, M. T. (2008) IL23 inhibits osteoclastogenesis indirectly through lymphocytes and is required for the maintenance of bone mass in mice. J. Immunol. 181, 5720-5729.

Rawlings, J. S., Rosler, K. M. and Harrison, D. A. (2004) The JAK/ STAT signaling pathway. J. Cell Sci. 117, 1281-1283.

Richards, C. D., Langdon, C., Deschamps, P., Pennica, D. and Shaughnessy, S. G. (2000) Stimulation of osteoclast differentiation in vitro by mouse oncostatin $\mathrm{M}$, leukaemia inhibitory factor, cardiotrophin-1 and interleukin 6: synergy with dexamethasone. $\mathrm{Cy}$ tokine 12, 613-621.

Sato, M., Grasser, W., Endo, N., Akins, R., Simmons, H., Thompson, D. D., Golub, E. and Rodan, G. A. (1991) Bisphosphonate action. Alendronate localization in rat bone and effects on osteoclast ultrastructure. J. Clin. Invest. 88, 2095-2105.

Shuai, K. (2000) Modulation of STAT signaling by STAT-interacting proteins. Oncogene 19, 2638-2644

Shuai, K. and Liu, B. (2003) Regulation of JAK-STAT signalling in the immune system. Nat. Rev. Immunol. 3, 900-911.

Stark, G. R., Kerr, I. M., Williams, B. R., Silverman, R. H. and Schreiber, R. D. (1998) How cells respond to interferons. Annu. Rev. Biochem. 67, 227-264.

Takayanagi, H., Kim, S., Koga, T., Nishina, H., Isshiki, M., Yoshida, H., Saiura, A., Isobe, M., Yokochi, T., Inoue, J., Wagner, E. F., Mak, T. W., Kodama, T. and Taniguchi, T. (2002a) Induction and activation of the transcription factor NFATc1 (NFAT2) integrate RANKL signaling in terminal differentiation of osteoclasts. Dev. Cell 3, 889-901.

Takayanagi, H., Kim, S., Matsuo, K., Suzuki, H., Suzuki, T., Sato, K., 
Yokochi, T., Oda, H., Nakamura, K., Ida, N., Wagner, E. F. and Taniguchi, T. (2002b) RANKL maintains bone homeostasis through cFos-dependent induction of interferon-beta. Nature 416, 744-749.

Takayanagi, H., Ogasawara, K., Hida, S., Chiba, T., Murata, S., Sato, K., Takaoka, A., Yokochi, T., Oda, H., Tanaka, K., Nakamura, K. and Taniguchi, T. (2000) T-cell-mediated regulation of osteoclastogenesis by signalling cross-talk between RANKL and IFN-gamma. Nature 408, 600-605

Tamura, T., Udagawa, N., Takahashi, N., Miyaura, C., Tanaka, S. Yamada, Y., Koishihara, Y., Ohsugi, Y., Kumaki, K. and Taga, T. (1993) Soluble interleukin-6 receptor triggers osteoclast formation by interleukin 6. Proc. Natl. Acad. Sci. USA 90, 11924-11928.

Teitelbaum, S. L. (2000) Bone resorption by osteoclasts. Science $\mathbf{2 8 9}$ 1504-1508.

Tondravi, M. M., McKercher, S. R., Anderson, K., Erdmann, J. M., Quiroz, M., Maki, R. and Teitelbaum, S. L. (1997) Osteopetrosis in mice lacking haematopoietic transcription factor PU.1. Nature 386, 81-84.

van Beek, E. R., Cohen, L. H., Leroy, I. M., Ebetino, F. H., Lowik, C W. and Papapoulos, S. E. (2003) Differentiating the mechanisms of antiresorptive action of nitrogen containing bisphosphonates. Bone 33, 805-811.

Walker, D. G. (1975) Spleen cells transmit osteopetrosis in mice. Science 190, 785-787.

Wei, S., Wang, M. W., Teitelbaum, S. L. and Ross, F. P. (2002) Interleukin-4 reversibly inhibits osteoclastogenesis via inhibition of NFkappa B and mitogen-activated protein kinase signaling. J. Biol. Chem. 277, 6622-6630.

Weitzmann, M. N., Cenci, S., Rifas, L., Brown, C. and Pacifici, R. (2000) Interleukin-7 stimulates osteoclast formation by up-regulating the T-cell production of soluble osteoclastogenic cytokines. Blood 96, 1873-1878.
Wu, H., Xu, G. and Li, Y. P. (2009) Atp6v0d2 is an essential component of the osteoclast-specific proton pump that mediates extracellular acidification in bone resorption. J. Bone Miner Res. 24, 871-885.

Yagi, M., Miyamoto, T., Sawatani, Y., Iwamoto, K., Hosogane, N., Fujita, N., Morita, K., Ninomiya, K., Suzuki, T., Miyamoto, K., Oike, Y., Takeya, M., Toyama, Y. and Suda, T. (2005) DC-STAMP is essential for cell-cell fusion in osteoclasts and foreign body giant cells. $J$. Exp. Med. 202, 345-351.

Yamada, A., Takami, M., Kawawa, T., Yasuhara, R., Zhao, B., Mochizuki, A., Miyamoto, Y., Eto, T., Yasuda, H., Nakamichi, Y., Kim, N., Katagiri, T., Suda, T. and Kamijo, R. (2007) Interleukin-4 inhibition of osteoclast differentiation is stronger than that of interleukin-13 and they are equivalent for induction of osteoprotegerin production from osteoblasts. Immunology 120, 573-579.

Yasothan, U. and Kar, S. (2008) Osteoporosis: overview and pipeline. Nat. Rev. Drug Discov. 7, 725-726.

Yoshida, H., Hayashi, S., Kunisada, T., Ogawa, M., Nishikawa, S., Okamura, H., Sudo, T. and Shultz, L. D. (1990) The murine mutation osteopetrosis is in the coding region of the macrophage colony stimulating factor gene. Nature 345, 442-444.

Yoshitake, F., Itoh, S., Narita, H., Ishihara, K. and Ebisu, S. (2008) Interleukin- 6 directly inhibits osteoclast differentiation by suppressing receptor activator of NF-kappaB signaling pathways. J. Biol. Chem. 283, 11535-11540

Zhang, Z., Welte, T., Troiano, N., Maher, S. E., Fu, X. Y. and Bothwell, A. L. (2005) Osteoporosis with increased osteoclastogenesis in hematopoietic cell-specific STAT3-deficient mice. Biochem. Biophys. Res. Commun. 328, 800-807.

Zou, J., Presky, D. H., Wu, C. Y. and Gubler, U. (1997) Differential associations between the cytoplasmic regions of the interleukin-12 receptor subunits beta1 and beta2 and JAK kinases. J. Biol. Chem. 272, 6073-6077. 